\title{
The assembly of stellar haloes in massive Early-Type Galaxies
}

\author{
F. Buitrago ${ }^{1,2}$ \\ ${ }^{1}$ Instituto de Astrofísica e Ciências do Espaço, Universidade de Lisboa, OAL, Tapada da \\ Ajuda, PT1349-018 Lisbon, Portugal \\ email: fbuitrago@oal.ul.pt \\ ${ }^{2}$ Departamento de Física, Faculdade de Ciências, Universidade de Lisboa, Edifício C8, Campo \\ Grande, PT1749-016 Lisbon, Portugal
}

\begin{abstract}
Massive $\left(\mathrm{M}_{\text {stellar }} \geqslant 5 \times 10^{10} \mathrm{M}_{\odot}\right)$ Early-Type Galaxies (ETGs) must build an outer stellar envelope over cosmic time in order to account for their remarkable size evolution. This is similar to what occurs to nearby Late-Type Galaxies (LTGs), which create their stellar haloes out of the debris of lower mass systems. We analysed the outer parts of massive ETGs at $z<1$ by exploiting the Hubble Ultra Deep Field imaging. These galaxies store 10-30\% of their stellar mass at distances $10<\mathrm{R} / \mathrm{kpc}<50$, in contrast to the low percentages $(<5 \%)$ found for LTGs. We find evidence for a progressive outskirt development with redshift driven solely via merging.
\end{abstract}

Keywords. galaxies: elliptical and lenticular, cD, galaxies: evolution, galaxies: halos

\section{Introduction}

Massive galaxies, especially ETGs, have changed dramatically their observational properties over cosmic time, in particular their sizes (Buitrago et al. 2008), morphologies (Buitrago et al. 2013), star formation rates (Pérez-González et al. 2008) and kinematics (Buitrago et al. 2014). To explain this transformation, it is thought that minor merging plays a key role in this process (Hopkins et al. 2009). These galaxies undergo continuous bombardment of minor satellites and thus creating an outer envelope via merging. - the so-called inside-out evolution, e.g. Oser et al. (2010)-.

Two questions arise: are these stellar envelopes/haloes similar to the ones created by late-type galaxies in the local Universe out of the debris of lower mass systems? Is it possible to catch individual massive galaxies in the very moment of forming these outskirts? Proving this galactic assembly beyond the nearby Universe is very challenging, due to the inherent faintness of these galaxy's outer parts and because of cosmological dimming diminishing the surface brightness as $(1+z)^{-3}$ (Giavalisco et al. 1996).

\section{Analysis}

To overcome these issues, we have used the deepest and best resolution optical/nearinfrared image of the Universe, the Hubble Ultra Deep Field, in its HUDF12 rendition (Koekemoer et al. 2012), which is the only version whose careful data reduction allow us to observe the extended outskirts of foreground galaxies. There, we have restricted ourselves to studying massive galaxies at redshifts $(z<1)$ where cosmological dimming is not yet severe $(\sim 2 \mathrm{mag})$. Interestingly, our final sample (6 objects) is composed of ETGs surrounded by a large number of potential satellites.

We made use of the 8 photometric bands available (ACS: F435W, F606W, F775W, F850LP; WFC3: F105W, F125W, F140W, F160W), which in all cases were carefully 


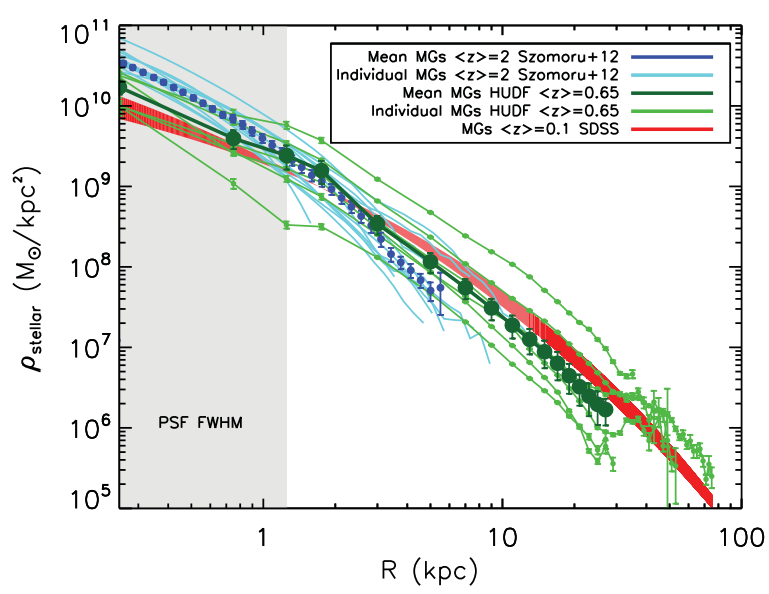

Figure 1. Circularized stellar mass density profiles for our sample, comparing them with similar mass local and high-z massive galaxies. Dark colors refer to mean profiles, whose slope in the outer parts become less steeper with $z$, thus pointing to the progressive build-up of stellar haloes.

reduced and sky-subtracted. We generously mask all galactic neighbours utilizing an automatic + manual algorithm. Then we derived observed and convolved surface brightness profiles. Special care was taken to determine the best PSF for profile decomposition. In order to avoid producing any artificial red halo, we built a hybrid star for each filter by using TinyTim (Krist 1995) to its maximum extent and then replacing the core with a suitable high signal-to-noise observed star. By so doing, we are able to reach down to $31 \mathrm{mag} \operatorname{arcsec}^{-2}$ or $\sim 25$ effective radii in galactocentric distances. This is at the level of Local Universe observations, but this time at a striking median redshift of $\langle z\rangle=0.65$.

\section{Results}

Fig. 1 shows individual (light colors) and mean (dark colors) mass profiles for massive ETGs in the local Universe (SDSS; red), in our sample (HUDF12; green) and at high-z (Szomoru et al. (2012); blue). The mean profiles' slope of the outer parts become less steeper with redshift, thus pointing to the progressive build-up of the galactic envelopes. To highlight this effect, we have integrated these mass mean profiles at $10<\mathrm{R} / \mathrm{kpc}<$ 50 , where our information is more robust and we only need to extrapolate the results from Szomoru's sample. We find that $3.5 \%$ of the galaxy mass is enclosed at these galactocentric distances for high-z case $(\langle z\rangle=2)$, the fraction is $22.6 \%$ at $\langle z\rangle=0.65$ and $28.7 \%$ at $\langle z\rangle=0.1$. Even though the total stellar mass for the three mean mass profiles is similar $\left(\sim 8 \times 10^{10} \mathrm{M}_{\odot}\right)$, the mass profiles of massive high-z ETGs are intrinsically different than their lower redshift counterparts.

Fig. 2 displays the mass fractions between 10 and $50 \mathrm{kpc}$ in the Cooper et al. (2013) simulations for both ETGs (red line) and LTGs (blue line) at $z=0$. We also provide the 16- and 84- quartile uncertainties (corresponding dashed lines) and the results for higher redshifts (yellow and pink lines; noisy because of the low number statistics). The green points are the galaxies in our sample, and are slightly below the local relation as a result of their redshifts (median $\langle z\rangle=0.65$ ). Our 10 and $50 \mathrm{kpc}$ boundaries represent the interval where we are dominated by accreted mass and confident with our color and mass determinations, and thus in good position to understand the relative importance of the galaxies' stellar haloes. There is a remarkable difference between ETGs and LTGs, where the former host more massive haloes $(>10 \%$ in mass) than the later $(<10 \%)$, 


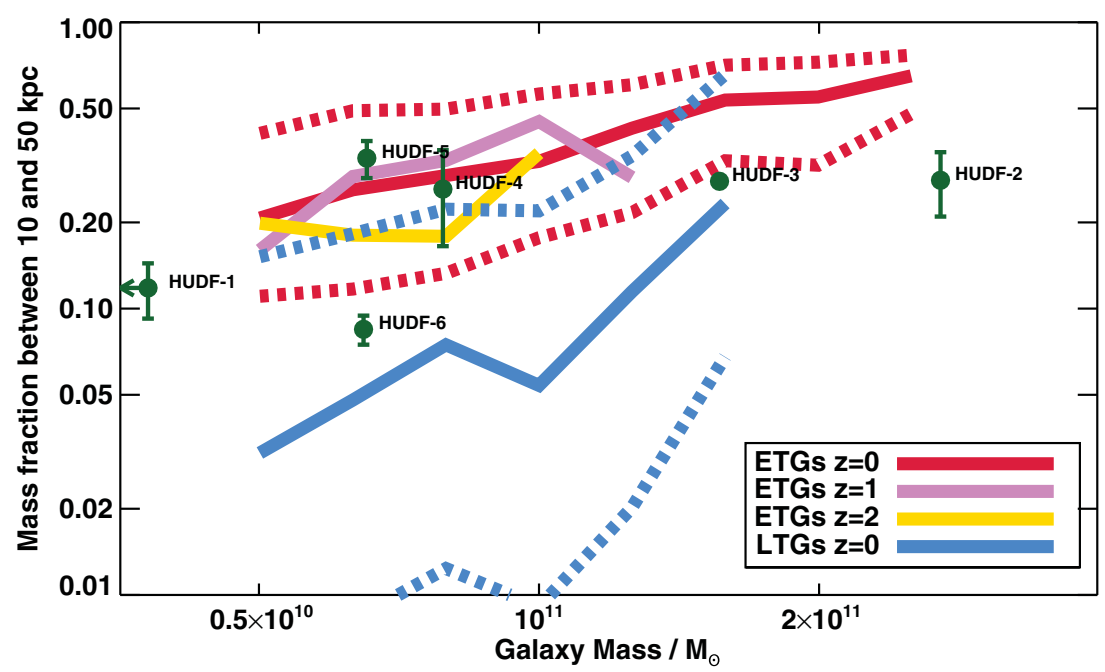

Figure 2. Mass fraction at $10<\mathrm{R} / \mathrm{kpc}<50$ for Early- and Late-Type Galaxies in Cooper et al. (2013) simulations, adding the 6 massive ETGs in our sample. Dashed lines indicate the scatter in the local relation, while high-z lines do not have enough number statistics. In summary, $10 \%$ in mass is the boundary between haloes belonging to either Early or Late-Type Galaxies.

with ETGs having typically $2-3 \times$ more mass stored in their outer parts than reported LTGs (see Trujillo et al. 2015 and references therein).

The reader can find more information about this work in Buitrago et al. (2016).

\section{References}

Buitrago, F., Trujillo, I., et al. 2008, ApJL, 687, L61

Buitrago, F., Trujillo, I., et al. 2013, MNRAS, 428, 1460

Buitrago, F., Conselice, C. J., et al. 2014, MNRAS, 439, 1494

Buitrago, F., Trujillo, I., et al. 2016, arXiv: 1602.01846

Cooper, A. P., D'Souza, R., et al. 2013, MNRAS, 434, 3348

Giavalisco, M., Livio, M., et al. 1996, AJ, 112, 369

Hopkins, P. F., Bundy, K., et al. 2009, MNRAS, 398, 898

Koekemoer, A. M., Ellis, R. S., et al. 2012, ApJS, 209, 3

Krist, J. 1995, ASP-CS, 77, 349

Oser, L., Ostriker, J. P., et al. 2010, ApJ, 725, 2312

Pérez-González, P. G., Trujillo, I., et al. 2008, ApJ, 687, 50

Szomoru, D., Franx M., et al. 2012, ApJ, 749, 121

Trujillo, I. \& Fliri, J. 2015, ApJ, 823, 123 